\title{
Cosmological Evolution Across Phantom Crossing and the Nature of the Horizon
}

\author{
Subenoy Chakraborty*, Nairwita Mazumder ${ }^{\dagger}$, Ritabrata Biswas ${ }^{\ddagger}$. \\ ${ }^{1}$ Department of Mathematics, Jadavpur University, Kolkata-32, India.
}

(Dated: November 1, 2018)

\begin{abstract}
In standard cosmology, with the evolution of the universe, the matter density and thermodynamic pressure gradually decreases. Also in course of evolution, the matter in the universe obeys (or violates) some restrictions or energy conditions. If the matter distribution obeys strong energy condition (SEC), the universe is in a decelerating phase while violation of SEC indicates an accelerated expansion of the universe. In the period of accelerated expansion the matter may be either of quintessence nature or of phantom nature depending on the fulfilment of the weak energy condition (WEC) or violation of it. As recent observational evidences demand that the universe is going through an accelerated expansion so mater should be either quintessence or phantom in nature. In the present work we study the evolution of the universe through the phantom barrier (i.e. the dividing line between the quintessence and phantom era) and examine how apparent and event horizon change across the barrier. Finally, we investigate the possibility of occurrence of any singularity in phantom era.
\end{abstract}

Keywords: Horizons, Phantom Barrier, Cosmological Evolution.

PACS numbers: $98.80 . \mathrm{Cq}, 98.80 . \mathrm{Bp}, 98.80 . \mathrm{Jk}$

\section{INTRODUCTION}

The universe at present should have a phase of deceleration in the context of standard cosmology. But recent observational evidences from the discovery of 16 type Ia Supernova (SNIa) by Riess et al [1] (there are other observations namely WMAP [2], SDSS [3] and X-ray [4]) using the Hubble Telescope has provided a distinct scenario. Contrary to the standard cosmological predictions it has been speculated that the universe must be in an accelerating era instead of a decelerating phase. To incorporate this accelerated expansion, attempts have been made to modify Einstein Equations [5] either from the left hand side (i.e. the geometry) or from the right hand side (i.e. the matter itself) if not both. Modification of the geometry indicates introduction of some modified gravity theory $(\mathrm{f}(\mathrm{R})$ gravity, Brane scenario etc) while change in the matter part indicates inclusion of some unknown kind of matters having large negative pressure so that SEC $(\rho+3 p>0)$ is violated. Such an unknown matter is known as dark energy(DE).

In literature, there are various $\mathrm{DE}$ model to match with observational data. The simplest model representing $\mathrm{DE}$ is the Cosmological Constant which was introduced by Einstein himself, surprisingly many years before the starting of DE craze. However, this model of DE is not very popular due to many inherent drawbacks (for example fine tuning problem [6]). The other candidates for DE are variable cosmological constant [7], the canonical scalar field [8] (quintessence field), scalar field with negative kinetic energy (phantom field) [9] or a quintom field [10] (a unified model of quintessence and phantom field). Further a combined effort of quantum field theory and gravity leads to speculate some nature of DE and is known as holographic dark energy (HDE) model [11].

The critical boundary where universe make a transition from quintessence era to the phantom era is known as phantom divide line or phantom crossing. In present work, we examine the consequences happened when universe leaves quintessence era and enters into the phantom era. We analyze the behavior of the horizons (apparent and event) across the phantom barrier and investigate any possible future singularity $[12,13]$.

\footnotetext{
*schakraborty@math.jdvu.ac.in

$\dagger$ nairwita15@gmail.com

$\ddagger$ biswas.ritabrata@gmail.com
} 


\section{THE EVOLUTION OF THE UNIVERSE: BASIC EQUATIONS}

For simplicity let us start with homogeneous and isotropic model of the universe (namely FriedmannRobertson-Walker(FRW) model), having line element

$$
\begin{gathered}
d s^{2}=-d t^{2}+a^{2}(t)\left[\frac{d r^{2}}{1-k r^{2}}+r^{2} d \Omega^{2}\right] \\
=h_{a b} d x^{a} d x^{b}+R^{2} d \Omega^{2}
\end{gathered}
$$

where

$$
h_{a b}=\operatorname{diag}\left(-1, \frac{a^{2}}{1-k r^{2}}\right) \quad, \quad\left(a, b=0,1 \text { with } x^{0}=t, x^{1}=r\right)
$$

and

$$
d \Omega^{2}=d \theta^{2}+\sin ^{2} \theta d \phi^{2} \text { is the metric on unit two sphere. }
$$

$R=a r$ is the radius of the sphere(area-radius), 'a' is the scale factor and $k=0, \pm 1$ stands for flat, closed and open model of our universe respectively.

The matter is chosen as a perfect fluid with energy momentum tensor

$$
T_{\mu \nu}=(\rho+p) u_{\mu} u_{\nu}-p g_{\mu \nu}
$$

and Einstein field equations are

$$
\begin{gathered}
H^{2}+\frac{k}{a^{2}}=\frac{8 \pi G}{3} \rho \\
\dot{H}-\frac{k}{a^{2}}=-4 \pi G(\rho+p)
\end{gathered}
$$

and the energy conservation equation is

$$
\dot{\rho}+3 H(\rho+p)=0
$$

Combining (3) and (4) we get,

$$
\dot{H}+H^{2}=\frac{\ddot{a}}{a}=-\frac{4 \pi G}{3}(\rho+3 p)
$$

The dynamical apparent horizon which is essentially the marginally trapped surface with vanishing expansion, is defined as a sphere of radius $R=R_{A}$ such that

$$
h^{a b} \partial_{a} R \partial_{b} R=0
$$

which on simplification gives

$$
R_{A}=\frac{1}{\sqrt{H^{2}+\frac{k}{a^{2}}}}
$$

The event horizon on the other hand is defined as [14]

$$
\begin{array}{cc}
R_{E}=-a \sinh (\tau) & k=-1 \\
R_{E}=-a \tau & k=0
\end{array}
$$




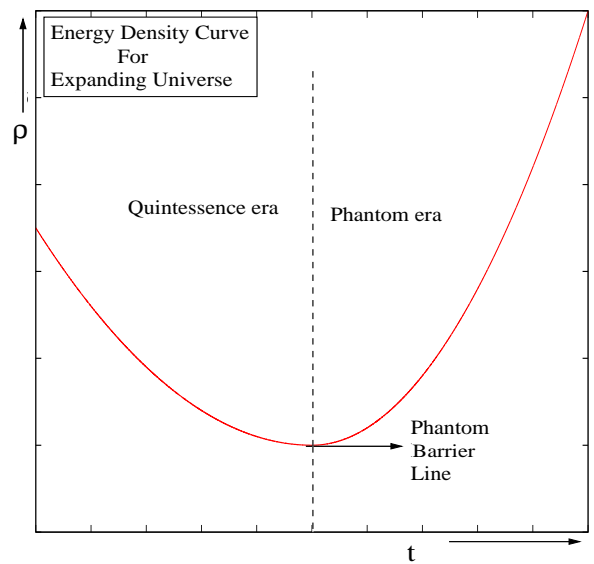

Fig.1

Fig.I represents the variation of energy density with the evolution of the universe in an expanding model. The dotted vertical line denotes the phantom divide or phantom barrier line.

$$
R_{E}=-a \sin (\tau) \quad k=+1
$$

where $\tau$ is the usual conformal time defined as

$$
\tau=-\int_{t}^{\infty} \frac{d t}{a(t)} \quad|\tau|<\infty
$$

Note that if $|\tau|=\infty$, event horizon does not exist. Also the Hubble horizon is given by

$$
R_{H}=\frac{1}{H}
$$

The time variation of the horizon radii are given by

$$
\begin{gathered}
\dot{R}_{A}=-H\left(\dot{H}-\frac{k}{a^{2}}\right) R_{A}^{3} \\
\dot{R}_{E}=H R_{E}-\sqrt{1-\frac{k}{a^{2}} R_{E}^{2}} \\
\dot{R}_{H}=-\frac{\dot{H}}{H^{2}}
\end{gathered}
$$

One may note that the expression for $\dot{R_{E}}$ given in references [14] and [15] are true only for $k=0$. So the theorems are valid for flat case only. However, from eq.(13) we see that $R_{E}$ is an increasing or decreasing function of time that depends only on whether $R_{E}>$ or $\left\langle R_{A^{-}}\right.$it does not depend on the nature of the matter involved. 




Fig.II represents the variation of energy density in an contracting model of the universe in phantom era.

\section{A. Discussion on the evolution of the Universe and the two horizons:}

We shall now discuss the evolution of the universe both in Quintessence and Phantom eras. Also the behavior of the horizons will be studied in these two eras.

From the conservation equation (5) we see that in Quintessence era $\rho$ is monotonic decreasing which reaches a local minima at the phantom crossing and increases again with the evolution of the universe as shown in Fig I. So the matter density has some short of bouncing behavior at the phantom crossing. However, if the universe starts contracting in phantom era (i.e., $H<0$ ) then conservation equation demands $\rho$ should still decreases in the phantom era and there is a point of inflexion at the phantom barrier as shown in Fig.II . For both the possibilities in phantom era $\rho$ has peculiar behavior when matter is exotic in nature (i.e., $\rho+p<0$ ). In the first case when universe is expanding $\rho$ also increases in the phantom era indicating some matter creation phenomena (of unknown nature) during that epoch. On the other hand, when universe starts contraction in the phantom era, $\rho$ still decreases, indicating destruction of mass in that era.

From the above equation (13) we note that $\dot{R}_{E}>0$ demands $R_{E}>R_{A}$ which is physically justified from the very definition of the horizons. Using the field equation (4) in the equation (12) we see that

$$
\begin{array}{r}
\dot{R}_{A}>0 \text { in quintessence era } \\
\quad<0 \text { in phantom era. }
\end{array}
$$

Thus both the horizons (as well as the Hubble horizon) increases in the quintessence era. In the phantom era, if the universe continue its expansion then $R_{A}$ gradually decreases and if we assume $R_{E}$ also decreases then it decreases more sharply than $R_{A}$ maintaining the restriction $R_{E}<R_{A}$ as shown in Fig III. The theorem given in Ref.[15] states that $\dot{R}_{E}<0$ is possible if universe blows up at finite time, indicating a possibility of a big rip singularity in phantom era.

On the other hand if $R_{E}$ still remain expanding in phantom era (see Fig IV.) while $R_{A}$ decreases then there is a possibility of naked singularity in future.

However, if the universe contracts in the phantom era then $\dot{R}_{E}<0$ and $\dot{R}_{A}>0$, there is a strange situation and it is not physically acceptable.

In this connection it is worthy to mention that if the matter is in the form of HDE then [16] 


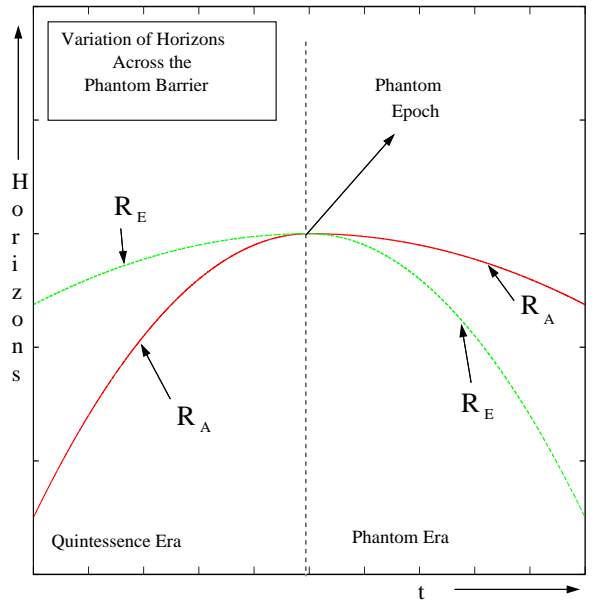

Fig.III

Fig.III represents variation of event horizon and the apparent horizon respectively in an expanding universe model. The dotted vertical line again denotes the phantom divide line. As the previous diagrams left side of which is denoting the quintessence era whereas the right hand side represents the phantom era.

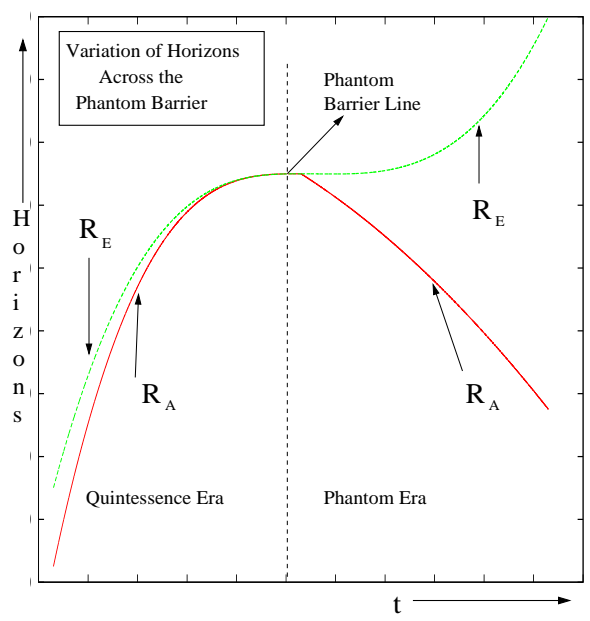

Fig.IV

Like Fig.III, Fig.IV also represents two curves showing the variation of event horizon and apparent horizon. The dotted vertical line denotes the phantom divide line. The left side of which is denoting the quintessence era whereas the right hand side represents the phantom era.

$$
\dot{R}_{E}=\frac{3}{2} R_{E} H\left(1+\omega_{D}\right)
$$

So in the phantom era if the universe expands then $\dot{R}_{E}<0$ so only Fig.III is possible. Further, if universe collapses in phantom era then both $R_{E}$ and $R_{A}$ increases and it is physically unacceptable. Finally we say that HDE has some distinct features in phantom era as compared to other fluids.

For future work, it will be interesting to explain the particle creation in the phantom era with the expansion of the universe and possibly the mechanism of particle creation may remove the possible future singularity.

\section{Acknowledgement :}


RB wants to thank West Bengal State Government for awarding JRF. NM wants to thank CSIR, India for awarding JRF. All the authors are thankful to IUCAA, Pune as this work has been done during a visit.

\section{REFERENCES}

[1] Riess A. G., et al., AstroPhys J. 607 665,(2004).

[2] C. L. Bennett et al., Astrophys. J. Suppl. 148, 1,(2003) .

[3] M. Tegmark et al. [SDSS Collaboration], Phys. Rev. D, 69, 103501, (2004) .

[4] S. W. Allen, et al., Mon. Not. Roy. Astron. Soc., 353, 457, (2004) .

[5] R.G. Cai and S.P. Kim, JHEP 02 050, (2005); Akbar M. and Cai R.G. Phys. Lett B 635 7,(2006) ; Lancoz C. ,Ann. Math. 39 842,(1938) ; S. Nojiri, S. Odintsov, arXiv: 0801.4843 [astro-ph]; S. Nojiri, S. Odintsov, arXiv: 0807.0685 [hep-th ]; S. Capozziello, IJMPD 11 483,(2002) ; S.M. Carroll, V. Duvvuri, M. Trodden and M.S. Turner Phys. Rev. D 68 043528,(2004) ; S. Nojiri and S.D. Odintsov, Phys. Rev. D 68 123512,(2003) ; S. Nojiri and S.D. Odintsov, Phys. Rev. D 74 086005, (2006).

[6] P. J. Steinhardt, itCritical Problems in Physics (1997), Princeton University Press.

[7] J. Sola and H. Stefancic, Phys. Lett. B 624147,(2005) ; J. Sola and H. Stefancic, Mod. Phys. Lett. A 21, 479,(2006) ; I. L. Shapiro and J. Sola, Phys. Lett. B 682105, (2009).

[8] B. Ratra and P. J. E. Peebles, Phys. Rev. D 37, 3406, (1988); C. Wetterich, Nucl. Phys. B 302, 668 (1988); A. R. Liddle and R. J. Scherrer, Phys. Rev. D 59, 023509 (1999); I. Zlatev, L. M. Wang and P. J. Steinhardt, Phys. Rev. Lett. 82, 896 (1999); Z. K. Guo, N. Ohta and Y. Z. Zhang, Mod. Phys. Lett. A 22, 883 (2007); S. Dutta, E. N. Saridakis and R. J. Scherrer, Phys. Rev. D 79, 103005 (2009).

[9] R. R. Caldwell, Phys. Lett. B 54523, (2002) ; R. R. Caldwell, M. Kamionkowski and N. N. Weinberg, Phys. Rev. Lett. 91071301, (2003) ; S. Nojiri and S. D. Odintsov, Phys. Lett. B 562147,(2003) ; V. K. Onemli and R. P. Woodard, Phys. Rev. D 70107301, (2004); M. R. Setare, J. Sadeghi, A. R. Amani, Phys. Lett. B 666, 288(2008); M. R. Setare and E. N. Saridakis, JCAP 0903,002(2009) ; E. N. Saridakis, Nucl. Phys. B 819, 6116(2009).

[10] B. Feng, X. L. Wang and X. M. Zhang, Phys. Lett. B 607, 35 (2005); Z. K. Guo, et al., Phys. Lett. B 608, 177(2005) ; M.-Z Li, B. Feng, X.-M Zhang, JCAP, 0512002,(2005); B. Feng, M. Li, Y.-S. Piao and X. Zhang, Phys. Lett. B 634101, (2006) ; M. R. Setare, Phys. Lett. B 641130,(2006) ; W. Zhao and Y. Zhang, Phys. Rev. D 73123509,(2006) ; M. R. Setare, J. Sadeghi, and A. R. Amani, Phys. Lett. B 660299, (2008) ; M. R. Setare and E. N. Saridakis, Phys. Lett. B 668177, (2008) ; M. R. Setare and E. N. Saridakis, JCAP $0809026,(2008)$; M. R. Setare and E. N. Saridakis, Int. J. Mod. Phys. D 18549,(2009) .

[11] V. Sahi , AIP Conf. Proc. 782166, (2005) ; [J. Phys. Conf. Ser. 31 115,(2006)] ; T. Padmanavan, Phys. Rept. 380 235,(2002) ; E.J. Copeland, M. Sami and S. Tsujikawa, IJMPD 15 1753,(2006) ; R. Durrer and R. Marteens, Gen. Rel. Grav. 40 301,(2008) ; S. Nojiri and S.D. Odintsov, Int. J. Geom. Meth. Mod. Phys. 4 115,(2007).

[12] Barrow J. D. , Class. Quantum Grav. 21L79, (2004).

[13] S.Nojiri and S.D.Odintsov, Phys. Rev. D 023003,72 (2005) .

[14] P.C.W. Davis, Class. Quantum Grav. 51349, (1998) .

[15] H.Mohseni Sadjadi, Phys. Rev. D 73063525, (2006) .

[16] N. Mazumder and S. Chakraborty, Gen.Rel.Grav.42 813, (2010). 
